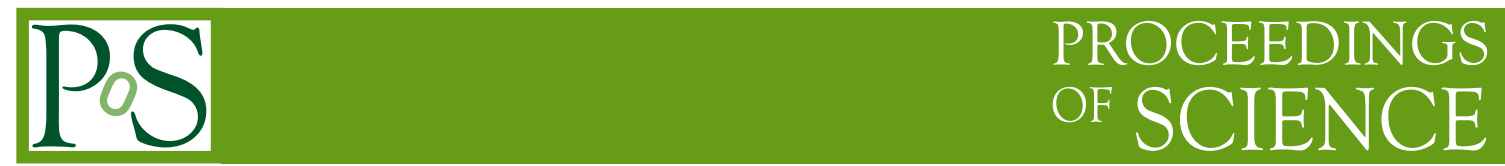

\title{
Magnetic monopoles in high temperature QCD
}

\author{
Alessio D'Alessandro* \\ Universita' di Genova \& INFN \\ E-mail: adales@ge.infn.it \\ Massimo D'Elia \\ Universita' di Genova \& INFN \\ E-mail: delia@ge.infn.it
}

We study the density and spatial distribution of thermal Abelian monopoles in the deconfined phase of SU(2) pure gauge theory: they display non-trivial interactions with a well defined continuum limit. The Maximal Abelian Gauge (MAG) has been chosen to perform the Abelian projection. Questions related to the choice of the Abelian projection, as well as possible directions for future studies, are discussed.

8th Conference Quark Confinement and the Hadron Spectrum September 1-6 2008

Mainz, Germany

${ }^{*}$ Speaker. 


\section{Introduction}

In the dual superconductor framework of the QCD vacuum [2] Abelian magnetic monopoles may explain color confi nement, which is related to the spontaneous breaking of a magnetic Abelian symmetry induced by monopole condensation. The magnetic condensate in the confi ned phase, as well as its disappearance at the deconfi ning transition, has been extensively observed on the lattice [3]. Magnetically charged particles have also been supposed to be relevant in explaining the physical properties of the Quark Gluon Plasma phase above the transition [ [ 5], such as the very low viscosity and diffusion coeffi cient [6] and the strongly interacting liquid-like nature [6, 6].

In Ref. [5] the magnetic component of the Quark Gluon Plasma (QGP) has been identifi ed with Abelian magnetic monopoles evaporating from the condensate and becoming thermal particles for $T>T_{c}$. A defi nition was given for their detection on the lattice in terms of non-trivially wrapped trajectories in the Euclidean time direction. In this work we apply this defi nition to fi nd the physical properties of these objects, such as their density and their correlation functions, in a wide temperature range above $T_{c}$. A full account of these results is presented in Ref. [1].

\section{The physical properties}

Simulations have been done for $S U(2)$ pure gauge for various lattice sizes and couplings. The physical scale has been determined as $a(\beta) \Lambda_{L}=R(\beta) \lambda(\beta)$, where $R$ is the two-loop $\beta$-function, while $\Lambda_{L}$ and the non-perturbative correction $\lambda(\beta)$ have been taken from Ref. [7].

We identify monopole currents with the usual De-Grand Toussaint construction [8], after Abelian projection in the Maximal Abelian Gauge. The gauge fi xing has been performed by maximizing, with a standard overrelaxation algorithm, the MAG functional

$$
F_{\mathrm{MAG}}=\sum_{\mu, x} \operatorname{Re} \operatorname{tr}\left[U_{\mu}(x) \sigma_{3} U_{\mu}^{\dagger}(x) \sigma_{3}\right]
$$

After that, monopole currents are defi ned $[\S]$ as $m_{\mu}=\frac{1}{2 \pi} \varepsilon_{\mu v \rho \sigma} \hat{\partial}_{v} \bar{\theta}_{\rho \sigma}$, where $\bar{\theta}_{\rho \sigma}$ is the compactifi ed part of the Abelian projected plaquette $\theta_{\sigma}$ and $m_{\mu}$ is an integer. Being closed $\left(\hat{\partial}_{\mu} m_{\mu}=0\right)$ these currents may be trivially or non-trivially wrapped along the time direction. The thermal monopole density [5], Q] is given in terms of the temporal winding number $N_{\text {wrap }}\left(m_{0}(\vec{x}, t)\right)$ of each time-directed current $m_{0}$ in $(\vec{x}, t)\left(V_{s}=\left(L_{s} a\right)^{3}\right.$ is the spatial volume):

$$
\rho=\frac{\left\langle\sum_{\vec{x}}\left|N_{\text {wrap }}\left(m_{0}(\vec{x}, t)\right)\right|\right\rangle}{V_{s}}
$$

Our results for different lattice spacings are reported in fi gure[1 (left). A nice scaling to the continuum limit can be observed. The curve does not fi t a simple $\rho \propto T^{3}$ behavior expected for free massless particles. A nice fit is instead obtained with $\rho=A T^{3} / \log ^{\alpha}\left(T / \Lambda_{\text {eff }}\right)$ with $A=0.48(4)$, $T_{c} / \Lambda_{\text {eff }}=2.48(3), \alpha=1.89(6)$ and a $\chi^{2} /$ d.o.f. of order 1 . At high temperatures also $\alpha=3$, which is the exponent expected [5, 10] from dimensional reduction and perturbative considerations $\left(\rho \sim\left(g^{2} T\right)^{3}\right)$ fi ts well. We conclude that interactions are important also at high temperatures.

The other quantity we look at is the monopole-(anti)monopole correlation function

$$
g_{++}(r)=\frac{<\rho^{+}(0) \rho^{+}(r)>}{<\rho^{+}>^{2}} \quad g_{+-}(r)=\frac{<\rho^{+}(0) \rho^{-}(r)>}{<\rho^{+}><\rho^{-}>}
$$



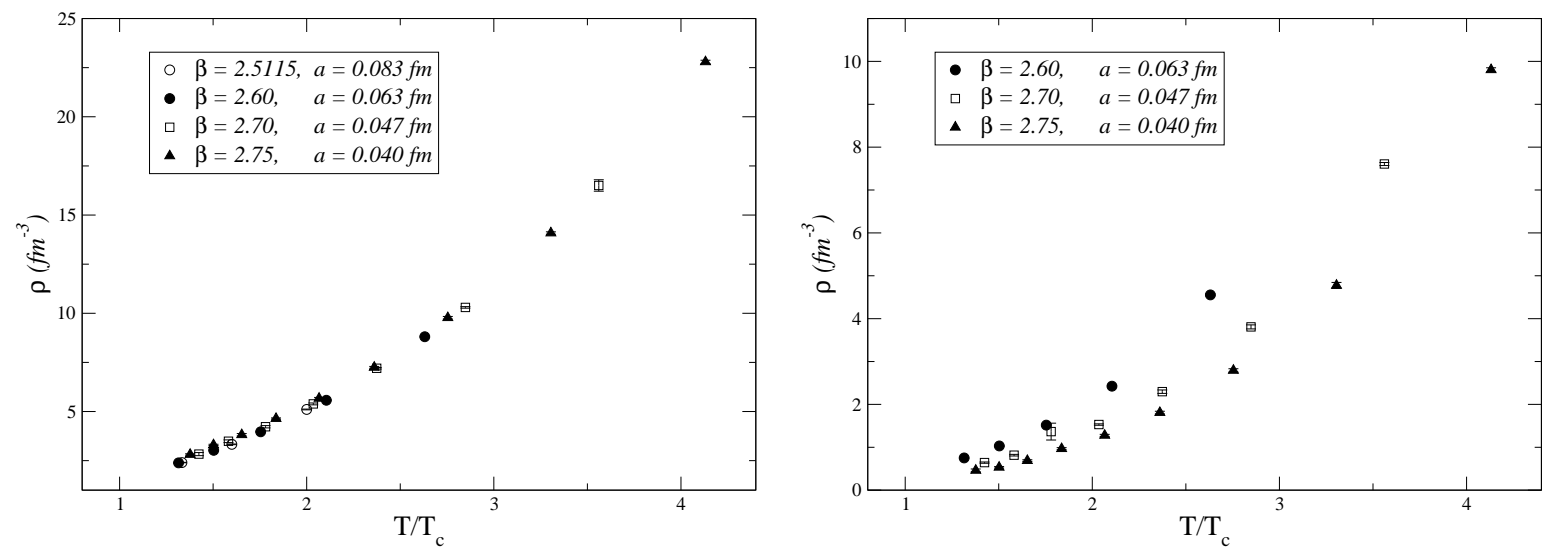

Figure 1: Monopole density with MAG gauge fixing from thermalized configurations [left] and with MAG gauge fixing after Landau preconditioning [right]. Spatial lattice sizes and $\beta$ 's are respectively $\left(L_{s}, \beta\right)=$ $(24,2.5115),(32,2.6),(40,2.7),(48,2.75)$ while the temporal sizes range from $L_{t}=4$ to $L_{t}=L_{s} / 4$.

for the monopole-monopole (++) and monopole-antimonopole (+-) cases $\left(\rho^{+}\right.$and $\rho^{-}$are the densities of timelike trajectories wrapped in the positive and negative time direction at a fi xed time-slice). This quantity is the probability of fi nding a (anti)monopole at distance $r$ from a given monopole normalized by the same probability in an uncorrelated situation. For the free case $g(r)=1$, while $g(r)>1(g(r)<1)$ means that at a distance $r$ we have more (less) particles then in the free case due to attraction (repulsion). For a solid we expect a multiple peak structure corresponding to several shells while in a liquid-like situation we usually expect only one large bump. This is just the situation for the monopole-antimonopole case as we see in fi g. 2 (left), from which a liquid-like behavior is qualitatively evident, with stronger interaction at high $T$ [11], where the bump gets higher. In fi g. 2 (right) we see, from the depletion region in $g_{++}(r)$ at short distances, that monopoles repel monopoles while at this distance there are more antimonopoles than in the free case $(g(r)>1)$, that means attraction between monopoles and antimonopoles. Notice also the very good scaling of $g(r)$, which means that even the correlation function is a well defi ned physical quantity.

Data have been fi tted according to $g(r)=e^{-V(r) / T}$, where $V(r)=\alpha_{M} e^{-r / \lambda} / r$ is a screened Coulomb potential, obtaining $\lambda \sim 0.1 \mathrm{fm}$. Further analysis [11] has led to an estimate of the plasma parameter $\Gamma=\alpha_{M}(4 \pi \rho / 3)^{1 / 3} / T(\sim$ interaction/kinetic energy) between 2 and 4.5 , which is a quantitative evidence for a liquid-like (nontrivial) interaction.

\section{The gauge dependence problem}

The defi nition of thermal monopoles, being based on the Abelian projection, is naturally subject to gauge dependence, or more precisely to dependence on the gauge where the Abelian projection is performed. That can be dramatic: for instance in Landau gauge wrapping trajectories are practically absent [1] at any $T$. More than that, even within the same Abelian projection Gribov copy ambiguities (due to the existence of multiple local maxima for the gauge functional) may lead to systematic effects. For example if we start the maximization of the MAG functional from a Landau gauge fi xed instead of a random thermalized confi guration, we fi nd a different result (fi . $1_{1}$ 

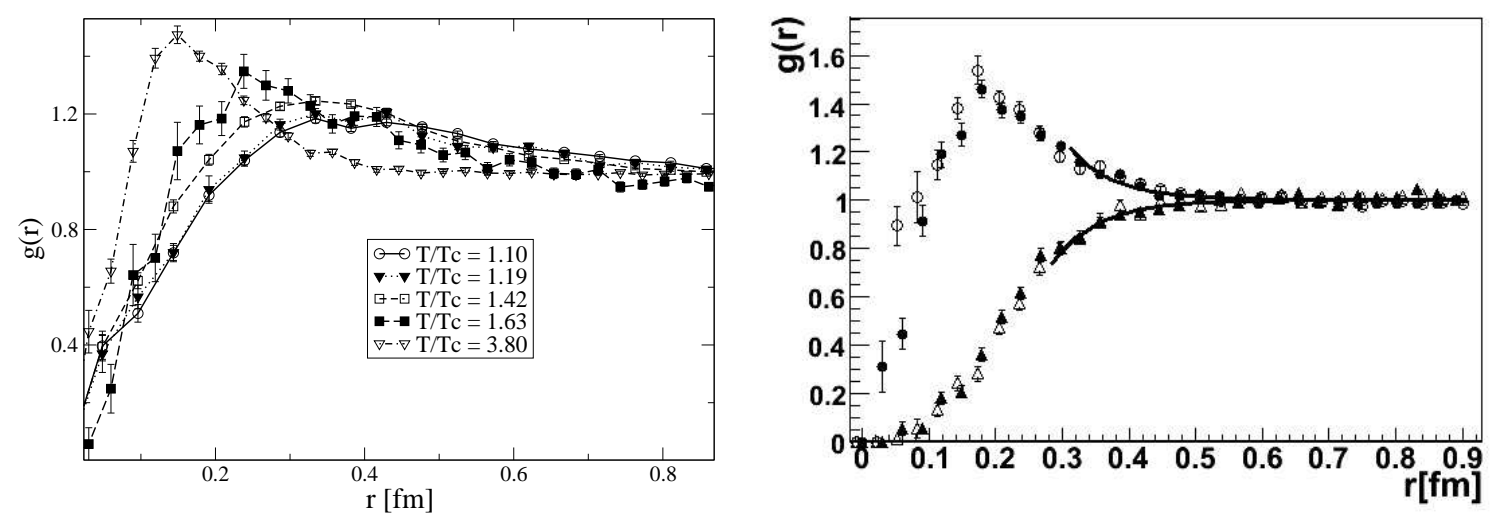

Figure 2: $g_{+-}(r)$ for $\beta=2.70$ and $L_{s}^{3} \times L_{t}=64^{3} \times 8$ at different $T / T_{c}$ [left]; $g_{+-}(r)$ (circles) compared with $g_{++}(r)$ (triangles), for $40^{3} \times 5$ at $\beta=2.70$ (empty markers) and $64^{3} \times 8$ at $\beta=2.86$ (full markers): both lattices correspond to the same $T / T_{c} \sim 2.85$ [right].

(right)) and -above all- the scaling is lost. Further investigations should be made by looking for the global maximum of the MAG functional via better algorithms, like simulated annealing [12].

Regarding the dependence on the gauge chosen for Abelian projection, one could try to look at different defi nitions of monopoles. One possibility is the gauge independent defi nition given in Ref. [13], where a partial gauge fi xing $S U(2)_{G} \times S U(2) / U(1)_{G^{\prime}} \rightarrow S U(2)_{G=G^{\prime}}$ is done maximizing

$$
F_{n M A G}=\sum_{x, \mu} \operatorname{Tr}\left(\left(\vec{\sigma} \cdot \vec{n}^{G^{\prime}}(x)\right) U_{\mu}^{G}(x)\left(\vec{\sigma} \cdot \vec{n}^{G^{\prime}}(x+\mu)\right) U_{\mu}^{G^{\dagger}}(x)\right)
$$

The adjoint color fi eld $\vec{n}$ (a unit vector) is linked to $U_{\mu}(x)$ via $V_{\mu}(x) \propto U_{\mu}(x)+(\vec{\sigma} \cdot \vec{n}(x)) U_{\mu}(x)(\vec{\sigma}$. $\vec{n}(x+\mu))$, where $V_{\mu}(x)$ is the unitarized $\vec{n}$-proportional part in the CFN [13] Hodge decomposition of the gauge fi eld $A_{\mu}$. Monopoles are then defi ned by applying the usual De Grand - Toussaint defi nition on the phase of $S U(2)$ gauge invariant plaquettes

$$
\theta_{\mu \nu}(x)=\arg \left[\operatorname{Tr} \frac{1+\vec{\sigma} \cdot \vec{n}(x)}{\operatorname{Tr}(1)} V_{\mu}(x) V_{v}(x+\mu) V_{\mu}^{\dagger}(x+v) V_{v}^{\dagger}(x)\right]_{\bmod 2 \pi}
$$

As this defi nition does not depend on simultaneous $S U(2)$ transformations $G(x)(\vec{\sigma} \cdot \vec{n}(x)) G^{\dagger}(x)$ and $G(x) V_{\mu}(x) G^{\dagger}(x+\mu)$ we are free to choose $\vec{\sigma} \cdot \vec{n}(x)=\sigma_{3}$ everywhere: in this way $F_{n M A G}=$ $F_{M A G}$ and $V_{\mu}(x)$ is the diagonal part of $U_{\mu}(x)$ selected in the same way as in the conventional Abelian projection $V_{\mu}(x)=U_{\mu}^{\text {diag }}=\operatorname{diag}\left(U_{1}^{1}, U_{2}^{2}\right)$ (unitarized); fi nally the projector $\operatorname{Tr}\left(\frac{1+\sigma_{3}}{\operatorname{Tr}(1)} \ldots\right)$ in 3.2 takes out the diagonal phase of the plaquette $\exp \left(i \theta_{\mu \nu} \sigma_{3}\right) \rightarrow \exp \left(i \theta_{\mu \nu}\right)$. The prescription in Ref. [13] with nMAG applied to 3.2 is then completely equivalent, by construction, to the usual Abelian monopole defi nition based on Abelian projection in the MAG gauge: therefore it does not provide, at least for gauge group $S U(2)$, an independent defi nition useful for further studies. However in this respect the nMAG formalism, based on theoretical evidences for Abelian dominace (see [13] and references therein), can be regarded as a justifi cation for adopting the MAG.

\section{Conclusions}

We presented a study of the density and correlation function of thermal Abelian monopoles 
in the deconfi ned phase of $S U(2)$ pure gauge theories: both of them show a good scaling to the continuum and confi rm the presence of non-trivial interactions among monopoles which could be relevant to QGP properties. Further analysis of the properties of wrapping trajectories is in progress and will give access to other properties of thermal monopoles, such as their physical mass.

Dependence on the gauge chosen for Abelian projection as well as Gribov copy effects remain at present a problem in the physical interpretation of thermal monopoles which needs clarifi cation. Different gauge invariant defi nitions [13] lead to monopoles which are identical to MAG monopoles and are therefore of no benefi $t$ in this respect, even if provide further inside in the MAG itself.

A different direction to be pursued in order to prove the physical nature of thermal monopoles is to study the excess of non-Abelian action around wrapped monopole trajectories [14].

\section{References}

[1] A. D’Alessandro and M. D’Elia, Nucl. Phys. B 799241 (2008).

[2] G. 't Hooft, in "High Energy Physics", EPS International Conference, Palermo 1975, ed. A. Zichichi; S. Mandelstam, Phys. Rept. 23, 245 (1976); G. Parisi, Phys. Lett. B60, 93 (1975).

[3] A. Di Giacomo, B. Lucini, L. Montesi, G. Paffuti, Phys. Rev. D 61, 034503 (2000), Phys. Rev. D 61, 034504 (2000); M. D’Elia, A. Di Giacomo, B. Lucini, G. Paffuti, C. Pica, Phys. Rev. D 71, 114502 (2005); M.N. Chernodub, M.I. Polikarpov and A.I. Veselov, Phys. Lett. B399, 267 (1997); P. Cea and L. Cosmai, JHEP 0111, 064 (2001); P. Cea, L. Cosmai and M. D’Elia, JHEP 0402, 018 (2004).

[4] C. P. Korthals Altes, hep-ph/0607154; J. Liao, E. Shuryak, Phys. Rev. C 75054907 (2007); Phys. Rev. C 77064905 (2008); M. N. Chernodub, K. Ishiguro, A. Nakamura, T. Sekido, T. Suzuki and V. I. Zakharov, PoS LAT2007, 174 (2007) [arXiv:0710.2547]; C. Ratti and E. Shuryak, arXiv:0811.4174 [hep-ph].

[5] M. N. Chernodub and V. I. Zakharov, Phys. Rev. Lett. 98, 082002 (2007); M. N. Chernodub and V. I. Zakharov, Proc. Nagoya 2006, arXiv:hep-ph/0702245.

[6] E. Shuryak, arXiv:0807.3033v2.

[7] J. Engels, F. Karsch, K. Redlich, Nucl. Phys. B 435, 295 (1995); J. Fingberg, U. Heller, F. Karsch, Nucl. Phys. B 392, 493 (1992).

[8] A. De Grand, D. Toussaint, Phys. Rev. D 222478 (1980).

[9] V.G. Bornyakov, V.K. Mitrjushkin and M. Muller-Preussker, Phys. Lett. B284, 99 (1992); S. Ejiri, Phys. Lett. B376, 163 (1996).

[10] P. Giovannangeli and C. P. Korthals Altes, Nucl. Phys. B 608, 203 (2001).

[11] J. Liao, E. Shuryak Phys. Rev. Lett. 101, 162302 (2008) [arXiv:0804.0255v3].

[12] V. G. Bornyakov, D. A. Komarov, M. I. Polikarpov and A. I. Veselov, arXiv:hep-lat/0210047.

[13] S. Ito, S. Kato, K. I. Kondo, T. Murakami, A. Shibata, T. Shinohara, Phys. Lett. B 645, 267 (2007).

[14] B. L. G. Bakker, M. N. Chernodub and M. I. Polikarpov, Phys. Rev. Lett. 80, 30 (1998);

B. L. G. Bakker, M. N. Chernodub, M. I. Polikarpov and A. I. Veselov, Phys. Lett. B 449, 267 (1999);

M. Chernodub, A. D'Alessandro, M. D'Elia and V. Zakharov, in progress, 